\title{
Selon $X$ en français médiéval : la construction des interprétations
}

\author{
Corinne Féron \\ Université du Maine-CELTED (EA 3474, Université de Metz) \\ corinneferon1@aol.com \\ Danielle Coltier \\ Université du Maine-CELTED (EA 3474, Université de Metz) \\ coltierdanielle@yahoo.fr
}

\section{Introduction}

En français contemporain, les groupes prépositionnels en selon sont susceptibles de recevoir diverses interprétations, que l'on peut regrouper en trois grandes catégories ; notre propos sera d'observer si ces diverses interprétations sont déjà attestées en français médiéval. Nous nous intéresserons tout particulièrement à l'interprétation que nous nommerons « origine », dans laquelle selon $X$ correspond à un commentaire du locuteur.

\subsection{Selon $X$ en français contemporain}

Nous avons tenté d'organiser les interprétations du groupe prépositionnel (Gprép.) selon $X$ en français contemporain (FC), en nous appuyant sur les articles de plusieurs dictionnaires (TLFi, NPR et Lexis), sur des travaux spécifiquement centrés sur la description de $\operatorname{selon}^{1}$ ou qui en donnent sporadiquement d'intéressants éléments de description (Molinier-Levrier 2000), enfin sur une étude de corpus.

Dans l'état actuel de notre travail, nous proposerons l'hypothèse suivante concernant le sens de selon: selon présente toujours le référent de son régime (désigné ici par $\mathrm{X}$ ) comme un point d'ancrage; à partir de ce sens, selon $X$, admet trois grandes interprétations : conformité, dépendance, origine, respectivement illustrées par :

1. Luc fabriquait effectivement du chocolat selon les normes.

2. Luc fabriquait effectivement du chocolat selon son humeur.

3. Luc fabriquait effectivement du chocolat (,) selon tous les témoins.

Dans les deux premières interprétations, selon $X$ contribue à la description du monde référentiel: le Gprép. est donc, dans la terminologie de Guimier 1996, endophrastique ${ }^{2}$; au contraire, dans l'interprétation « origine », selon $X$ est exophrastique.

Nous décrivons dans les sous-divisions suivantes chacune de ces interprétations.

\subsubsection{SELON 1 : « conformité »}

$\mathrm{X}$ est un point d'ancrage pour la description (qualification, classification, etc.) d'une action (il est alors un modèle pour l'action) ou pour l'évaluation (il est alors un critère pour l'évaluation, par ex. dans : une chambre selon mon goût, c'est une femme selon Dieu). Dans cette interprétation, selon $X$ est incident $^{3}$ à un segment, qu'on nommera Y, qui correspond à un constituant de la phrase - par ex. le verbe en (4), le verbe et son complément en (1) -, à un constituant de niveau inférieur (ex. 5) ou à la phrase (ex. 6) :

4. Il a agi selon mes ordres.

5. Il a trouvé une chambre selon son gout. 
6. Ici, on se marie le samedi, selon la tradition.

Le segment auquel selon $X$ est incident fait partie des présupposés de l'énoncé et le référent du syntagme nominal $(\mathrm{SN})$ régi est un « particulier ».

\subsubsection{SELON 2 : « dépendance »}

$\mathrm{X}$ est un point d'ancrage pour l'explication d'une variation (dont il constitue, souvent, le type de cause ou de raison). Comme dans l'interprétation précédente, selon $X$ est incident à un constituant de la phrase, à un constituant de rang inférieur ou à la phrase, et le contenu du segment auquel le Gprép. est incident fait partie des présupposés de l'énoncé. La différence majeure avec SELON 1 tient au fait que le SN régi ne réfère pas à un particulier, mais à une forme de pluralité, et ce, même quand le SN est au singulier ; dans (7), par exemple, le SN la couleur du fil renvoie à un pluriel de couleurs (chaque bobine est associée distributivement à une couleur de fil) :

7. Il eut à trier des bobines selon la couleur du fil, puis des sachets d'aiguilles (Robert Sabatier, David et Olivier, 1985, p. 356, dans Frantext).

\subsubsection{SELON 3 : " origine »}

$\mathrm{X}$ est un point d'ancrage pour l'énonciation par L (le locuteur-en-tant-que-tel) de Y - que ce segment Y soit une phrase (ex. 3), un constituant de la phrase (ex. 9), ou encore un élement placé en incidente ( $c f$. infra, ex. 15); on admettra que, dans tous les cas, Y correspond à un contenu propositionnel, $p$.

Dans cette interprétation « origine », nous distinguons plusieurs nuances interprétatives.

- SELON 3A. Selon $X$ spécifie un DOMAINE DE VALIDITE. Trois cas sont possibles :

- SELON 3A-1 «marqueurs d'opinion forte » (Borillo 2004 : 38). Le SN régi selon moi :

8. Selon moi, avec le temps je suis devenu de plus en plus idiot - c'est la vérité pure.

(C. Isherwood, Un homme au singulier, Point, 143)

9. Prétexte de sa visite : déposer ici dans des caisses tout ce qu'il avait pu soustraire aux huissiers. But inavoué mais véritable selon moi : s'épancher dans le giron d'Iris (François Dorin, Les vendanges tardives, 1907, p. 251, dans Frantext).

- SELON 3A-2 «rapport de parole ». Soit X réfère directement ou indirectement à un humain; $p$ est (montré comme) construit à partir d'un acte de parole de $\mathrm{X}^{4}$ (une situation d'énonciation est présupposée, dans laquelle $\mathrm{X}$ a été locuteur) ; on est dans des cas d'emploi de selon comme marqueur de Discours rapporté au sens de Authier-Revuz (1992 et 1993) :

10. Raymond, que rien ne surprend à l'accoutumée, en parle presque dévotieusement.

Lisant l'incompréhension dans mon regard, il entreprend de m'éclairer. Selon lui,

Deauville est la seule plage où l'on doive être vu au mois d'août !... (Albert Simonin,

Confessions d'un enfant de la Chapelle, 1977, p. 267 dans Frantext)

Soit $\mathrm{X}$ réfère - en référence directe - à un objet produit par le discours (légende, récit, rapport) et vu comme Locuteur :

11. Anthropoïde géant à face humaine, qui vivrait selon certaines légendes dans les hautes montagnes himalayennes. (Définition de Yéti dans le TLFi)

12. Charlotte était morte et à l'endroit de la Stalinka, selon le récit d'Alex Bond, on avait construit un stade. (Andreï Makine, Le testament français, 1995, p. 308, dans Frantext)

Le SN peut être constitué - outre d'un pronom personnel - d'un nom, propre (patronyme et prénom) ou commun, désignant un humain; il peut aussi s'agir d'un nom capable d'y référer indirectement (selon la police/Bonn), ou encore d'un nom, propre ou commun, désignant des objets produits par le discours 
(texte, mot, dire, Bible, Coran, Les Misérables, etc.) ; enfin de noms catégorisant ou qualifiant l'acte de dire (aveu, déclaration, rumeur, etc.)

Dans cette interprétation, $\mathrm{L}$, suivant les cas, prend en charge le contenu $p$, le rejette ou reste neutre.

- SELON 3A-3 « transfert ». X est interprété comme un univers de discours :

13. Selon Freud tu passes par un age de perturbation sexuelle ou l'homosexualité passagere est possible, dans quelques annees tu sauras reellement qui tu es, sinon ... (Internet, ponctuation et orthographe respectées).

Dans (13), X est en référence indirecte. Au moyen du SN Freud, L réfère non à l'individu Freud mais indirectement à la théorie psychanalytique de Freud). C'est L qui élabore le contenu $p$ à partir de la connaissance qu'il a et de la théorie, et de la situation de celui dont il parle (ici le référent du sujet du verbe de la phrase). Nous dirons que le locuteur « transfère ».

Cette interprétation présuppose l'existence d'un discours, discours qui est une théorie particulière, ou encore un corps de principes ou de préceptes. Interviennent aussi, dans l'interprétation, des connaissances extra-linguistiques : ainsi, dans (13), la connaissance par l'interprétant du fait que l'individu Freud n'a pas pu tenir de discours sur le référent de «tu»; la «traduction» de (13) dans les formes canoniques du discours rapporté se ferait au moyen d'un conditionnel pour le verbe de parole (Freud dirait/expliquerait que... $)^{5}$.

Dans cette nuance «transfert», p, nécessairement vrai dans l'univers de discours X, ne l'est pas nécessairement pour L, ni pour l'individu que désigne $\mathrm{X}$; par contre, aux yeux de $\mathrm{L}$, il existe une proposition générale (il y un âge où l'homosexualité passagère est possible) que l'énoncé actualise sur un cas particulier.

- SELON 3B « Mediatif ». Selon $X$ indique que $p$ est vrai soit dans un univers de croyance autre que l'univers de $j e$ en $\mathrm{t}_{\mathrm{o}}$, soit dans un univers de discours; $p$ est construit par L. On distinguera ici deux cas :

- SELON 3B-1 «opinion attribuée à un tiers» humain ou non, spécifique. Le SN régime de selon a le trait /animé/ ; $p$ est construit par L à partir d'un comportement autre que verbal de $\mathrm{X}$ :

14. Je vois à votre air que je passe bien vite selon vous, sur ces détails qui ont du sens (Camus, La chute, Pléiade, p. 1539)

15. Par ailleurs, sous prétexte d'améliorer l'équilibre nutritif de leur repas, ils ajoutent des légumes dans la terrine : forcément ça leur reviens [sic] moins cher! Pourtant le prix a augmenté. Les plus : c'est bon (selon mon chat). Les moins? C'est cher. (www.toluna.fr/Le_nec_plus_ultra-av-84275.html)

Dans (14) et (15), X réfère à l'univers de croyance auquel L attribue le contenu de $p$. Le contenu de $p$ est construit par L sur la base d'une activité, d'un comportement, d'une mimique de X, d'un acte, etc. (acte autre qu'un comportement verbal) qui est considéré comme un indice et qui est verbalisé explicitement dans le discours (ex. 14 : à votre air) ou non (ex. 15). Dans (15), en effet, où L juge des aliments pour animaux, le comportement de $\mathrm{X}$ est facilement reconstructible par l'interprétant. L, suivant les cas, prend $p$ en charge, le rejette ou reste neutre.

- SELON 3B-2 «mode d'accès à une information ». X ne désigne pas un animé, mais fait référence à un moyen d'accéder à de l'information - l'autopsie dans (16), l'horloge, dans (17), l'orthographe dans (18), le cheminement du crayon et donc les traces qu'il laisse dans (19), le costume dans (20) :

16. Au moment où il est mort - à 3 heures selon l'autopsie,$- \ldots$

17. Moi je ne mourrai pas encore. En effet, selon l'horloge il restait 5 minutes. Il regarda le cadran lumineux, fronça les sourcils et montra ses dents blanches à l'horloge en un rictus hideux, et s'il mourrait subitement empoisonné ? (Internet 2006)

18. Arrêtons de médire de ce pauvre [...], et disons que peut être, ayant arrêté l'école à ans selon ses dires, en maternelle 2 selon son orthographe... (phenixecarlate. (com/forums/viewtopic.php?p=34543) 
19. - Non, Léonard n'était pas seul. / - Il y avait au moins celui qui le photographiait. / - Celui-là et quelques autres. // Trois ou quatre selon le nouveau cheminement $d u$ petit feutre_rouge dans les profondeurs obscures de la vieille photo. Et peut-être d'autres, hors champ. (D. Pennac, Au bonheur des ogres, Folio, p. 201)

20. Le tombeau a vraisemblablement été exécuté un certain temps après la mort, en 1473, d'Olivier de la Chapelle. Selon le costume, la dalle a pu l'être dans les années 1490. (www.mobilier-de-france.org/mobilier)

$\mathrm{X}$ réfère, de façon directe ou non, à ce qui permet à $\mathrm{L}$ de construire $p ; p$ est ou non pris en charge par L. Le régime de selon est un nom d'activité (plus ou moins intellectuelle : calculs, observations), un nom qui présuppose une situation de recherche d'informations (les indices, les traces, les empreintes), ou dont le référent est un objet "fabriqué » (horloge, cadran solaire) qui fournit ou permet de construire des informations; il peut également s'agir de noms susceptibles de référer à des objets du monde qu'un individu peut «transformer » en indices pour construire des informations hypothétiques : le petit feutre dans (5), l'orthographe dans (6), le costume dans (7).

Voici, en résumé, les nuances interprétatives de SELON 3.

Tableau 1 : SELON 3

\begin{tabular}{|c|c|c|}
\hline & & Régime de la proposition \\
\hline \multirow{3}{*}{$\begin{array}{l}\text { SELON 3A : spécifie un } \\
\text { domaine de validité }\end{array}$} & $\begin{array}{l}\text { SELON 3A-1 : selon } \\
\text { moi « marqueur } \\
\text { d'opinion forte }\end{array}$ & $m o i$ \\
\hline & $\begin{array}{l}\text { SELON 3A-2 « rapport } \\
\text { de parole » }\end{array}$ & $\begin{array}{l}\text { - nom référant à un humain, directement (Selon } \\
\text { mon voisin...) ou indirectement (Selon Bonn...) } \\
\text { - nom désignant un objet produit par le discours } \\
\text { (Selon la Bible...) }\end{array}$ \\
\hline & $\begin{array}{l}\text { SELON 3A-3 } \\
\text { «transfert » }\end{array}$ & $\mathrm{X}$ est en référence indirecte (Selon Freud, tu es... ) \\
\hline \multirow{2}{*}{ SELON 3B : médiatif } & $\begin{array}{l}\text { SELON 3B-1 : « opinion } \\
\text { attribuée à un tiers » } \\
\text { humain ou non }\end{array}$ & N /animé/ \\
\hline & $\begin{array}{l}\text { SELON 3B-2 : « mode } \\
\text { d'accès à une } \\
\text { information » }\end{array}$ & $\begin{array}{l}\text { X désigne un moyen d'accéder à l'information } \\
\text { (Selon les empreintes...) }\end{array}$ \\
\hline
\end{tabular}

\subsection{Présentation de l'étude diachronique}

Les Gprép. selon X relevant de SELON 3 («origine ») sont, on l'a vu, exophrastiques : ils véhiculent un commentaire du locuteur, qui signale que l'énonciation de Y (élément auquel selon $X$ est incident) a, d'une façon ou d'une autre, X pour origine. Dans les autres interprétations (SELON 1 et SELON 2), le Gprép. est au contraire endophrastique : il participe à la création du sens référentiel.

Or, quand une même unité ou séquence peut avoir ces deux types d'emploi dans un état de langue donné, on observe, de façon générale, que l'emploi endophrastique est le plus ancien, l'emploi exophrastique n'apparaissant que progressivement ( $c f$. par ex. Combettes 1995 pour de toute(s) façon(s), en principe, à la rigueur; Traugott 1989 pour les adverbes modaux possibly, probably, evidently, apparently, obviously $^{6}$ ) : il s'agit là d'un cas de subjectification, définie comme un «pragmatic-semantic process 
whereby 'meanings become increasingly based in the speaker's subjective belief state/attitude toward the proposition', in other words, toward what the speaker is talking about » (Traugott $1995: 31$ ).

Dans le cas des Gprép. en selon, nous verrons (section 2) que cette succession des emplois dans le temps n'apparait pas clairement : si les toutes premières occurrences sont endophrastiques, les dictionnaires étymologiques et les dictionnaires de français médiéval mentionnent des exemples très anciens dans lesquels selon $X$ peut être interprété comme exophrastique.

On pourrait penser qu'il s'agit là d'un emploi hérité du latin; deux étymologies ont été proposées pour selon, mais que l'on retienne l'une ou l'autre, l'hypothèse en question n'est pas véritablement vérifiable : soit selon vient de *sublongum, forme à laquelle on attribue le sens de «le long de », mais qui n'est pas attestée; soit selon provient d'un croisement entre les prépositions son (ou sonc, issu de secundum) et $l_{\text {lonc }}^{7}$; si secundum préposition est bien attesté ${ }^{8}$, il ne s'agit pas, néanmoins, de l'étymon direct de selon.

Dès lors, notre objectif sera d'observer comment se construit l'interprétation SELON 3 en français médiéval; le travail sera mené à partir d'occurrences extraites de bases textuelles informatisées (section $3)$.

\section{Les interprétations de selon $X$ en français médiéval (1) : examen de dictionnaires}

\subsection{Dictionnaires étymologiques et rubriques étymologiques de dictionnaires généraux}

D'après les dictionnaires consultés ( $F E W, T L F i$ et $G L L F)$, les premières occurrences connues de selon (sous les graphies sulunc et selunc) figurent dans La vie de saint Alexis (milieu $\mathrm{XI}^{\mathrm{e}} \mathrm{s}$.) : l'une ne se trouve que dans le manuscrit L (vers 1120), l'autre dans deux manuscrits, $\mathrm{A}$ (à peu près contemporain de $\mathrm{L}$ ) et $\mathrm{P}$ (XIII s. $)^{9}$.

A partir des informations fournies par ces mêmes dictionnaires, on peut reconstituer la chronologie suivante pour les interprétations de selon préposition ${ }^{10}$ :

1. «Suivant, conformément à », dès La vie de saint Alexis.

2. «Suivant l'opinion de » (1174-1176).

3. « Le long de » (1181-90).

4. «En proportion de, dans la mesure de $\left(\mathrm{XII}^{\mathrm{e}}\right.$, avant 1188).

5. «En fonction de » (début XIII $\left.{ }^{\mathrm{e}}\right)$.

6. «Du fait de, en conséquence de » (1215).

7. «Si l'on se réfère à tel principe, à telle manière de penser et de juger, à tel texte ou à telle oeuvre » (v. 1370, Oresme).

8. «Relativement à, par rapport à » $\left(\mathrm{XIV}^{\mathrm{e}}-\mathrm{XV}^{\mathrm{e}}\right)$.

C'est donc SELON 1 ( « suivant, conformément à ») qui serait attesté en premier ; viendraient ensuite :

- SELON 3 (à quoi correspond la définition « suivant l'opinion de »), avec pour régime un SN /humain/; une autre interprétation relevant également de SELON 3 (« si l'on se réfère à tel principe, à telle manière de penser et de juger, à tel texte ou à telle oeuvre ») est datée du XIV ${ }^{\mathrm{e}} \mathrm{s}$. ;

- l'emploi dans le domaine spatial ( «le long de »);

- et enfin SELON 2 (« en proportion de, dans la mesure de »; « en fonction de »). 
Restent deux définitions, toutes deux fournies par le $F E W$ sans références précises : « du fait de, en conséquence de » et « relativement à, par rapport à »; la première pourrait se rattacher à SELON 2 et la seconde n'est pas, semble-t-il, sans rapport avec SELON 3 ; toutefois, en l'absence d'exemples, ces rapprochements ne peuvent être précisés.

L'interprétation SELON 3, avec un régime désignant un être humain, daterait donc ${ }^{11}$ des environs de 1175 ; voici l'exemple que cite le $T L F$ :

21. [1174-76] Guernes de Pont-Sainte-Maxence, Saint Thomas, éd. E. Walberg, v. 3420 : Qui voldreit clerement a la raisun guarder, / Mielz vient de basse gent e bon estre e munter / Que de halte gent estre e en enfer aler. / Les mains honestes menbres deit l'um plus honurer, / Sulunc l'apostle, e plus d'onur envoluper. (« ̀̀ bien considérer la raison, mieux vaut appartenir aux petites gens probes et s'élever, qu'appartenir à la haute société, et aller en enfer. Selon l'apôtre, on doit honorer davantage les membres les moins honnêtes, les entourer de plus d'honneur. » Trad. de J. Thomas, Louvain/Paris : Peeters, 2002, p. 207.]

Une interprétation en SELON 1 (conformité) pourrait être envisagée (moyennant la suppression de la virgule que l'éditeur a insérée devant la préposition); parallèlement à un exemple tel que (22) :

22. [c. 1218-1227] Gautier de Coincy, Les miracles de Notre Dame, éd. V. F. Koenig, t. 2, p. 70 : Don ne service ne presist / De benefice ne de rente, / Mais selonc Dieu sanz autre entente / Ses biens departoit li Dieu sers / As preudomes et as bons clers. [ «Il n'aurait jamais accepté de don ni de service en échange d'un bénéfice ou d'une rente, mais selon [les commandements de] Dieu, sans autre intention, ce serviteur de Dieu distribuait ce qu'il possédait aux hommes de bien et aux bons clercs. »]

sulunc l'apostle serait dans la dépendance de honurer et indiquerait le «modèle » selon lequel le procès doit se réaliser (le Gprép. serait alors endophrastique). L'objection majeure à cette interprétation tient au nom même qui est régime de selon: apostle n'est pas associé à des préceptes concernant la manière d'agir, mais défini comme «l'auteur », ou au moins l'origine d'un texte ${ }^{12}$, que la proposition « Les mains honestes menbres deit l'um plus honurer » reprend (non littéralement) en partie; nous avons donc sans doute un SELON 3A-2 « rapport de parole».

Le $T L F i$ cite par ailleurs, sous la définition «suivant, conformément à », un exemple (avec un régime désignant un texte) qui, d'après nous, pourrait également relever de SELON 3A-2 « rapport de parole », mais cette fois avec un régime référant à un objet produit par le discours :

23. [1121-1134] Philippe de Thaon, Bestiaire, éd. E. Walberg, v. 930 : Ço DIENT escriptures, / Furmiz at treis natures : / Il at tel naturete / Quant ist de sa fossete (...) / OËZ L'ALTRE nature / Sulunc sainte escripture : / Le grenet [ " petit grain »] que il at / En dous [«deux »] parz le fendrat ; / Issi fait cuintement [«prudemment »] / Qu'en iver faim nel prent. [«de telle manière qu'en hiver elle n'est pas surprise par la faim »]

Il s'agit ici de faire entendre ce que L'Ecriture sainte dit de « l'altre nature » de la fourmi.

\subsection{Dictionnaires de français médiéval}

Contrairement à l' $A N D$, dans lequel l'une des traductions de selon est « to judge by », le GD et le $T L$ ne donnent aucune définition correspondant à SELON 3 ; ils citent pourtant - sous "suivant, conformément à » pour le premier, sous « gemä $\beta$ » pour le second - des exemples interprétables comme des SELON 3 ou, au moins, pour lesquels cette interprétation peut être envisagée. L'un de ces exemples est antérieur à ceux que l'on a cités précédemment : il s'agit d'un extrait du Comput de Philippe de Thaon (que nous citons ici d'après l'éd. I. Short), dans lequel, comme dans l'exemple (21), selon permet au locuteur de renvoyer, dans son propre discours, au dire d'autrui :

24. [1119] Phillipe de Thaon, Comput, éd. I. Short, v. 287-288: Sulunc les clers devins / E sulunc les Latins / 'Nuit' est dit a 'nuisir' [ " 'nuit' vient de 'nuire' »] ; / E fait 


\begin{abstract}
la gent dormir / Le seir [« soir »] reposer, / Ki ne veient a ovrer [« qui n’y voient pas pour travaiiller »]; / Pur ço que n'unt lüur [«parce qu'ils n'ont pas de lumière »] / Cessent de lur labur, / Altrement perireient / E par travail murreient.
\end{abstract}

Les autres exemples relevant de SELON 3 sont également du type « rapport de parole », mais avec un régime désignant le résultat ou le produit d'une activité de discours ${ }^{13}$ : le conte, sainte escripture, l'escripture...

$\mathrm{Au}$ total, les emplois répertoriés dans les dictionnaires consultés suggèrent que dès l'ancien français, les interprétations de selon $X$ - mis à part les emplois dans le domaine spatial - pourraient être réparties dans les trois grandes catégories retenues pour le FC. En particulier, avec un régime désignant un animé humain (ex. 21 et 24) ou le résultat d'une activité de discours (ex. 23), une interprétation SELON 3 peut être construite, et ce dès la $1^{\text {ere }}$ moitié du XII $\mathrm{s}$.

\title{
3 Les interprétations de selon $X$ en français médiéval (2) : étude de corpus
}

\subsection{Présentation}

Notre étude de corpus portera sur les cas où le régime de selon réfère à un animé humain. On observera notamment les caractéristiques sémantiques et référentielles du régime de la préposition et les caractéristiques de l'énoncé (présence d'éléments suggérant une alternative ou une variation, énoncé à valeur générale ou spécifique, impliquant ou non une évaluation...).

Nos requêtes portent sur toutes les graphies de selon: selonc, selunc, solonc, etc. (nous n'avons pas relevé de formes en $-r$-, tels que soron, seron, seront, pourtant attestées en ancien français). Nous scindons l'étude en deux époques : ancien français (des premiers textes jusque 1329), moyen français (1330-1500) ; pour la première, nous utilisons les textes d'ancien français de la Base du français médiéval $(B F M)^{14}$ et les textes accessibles sur le site de $1^{\prime} A N D^{15}$; pour la seconde, la base textuelle du Dictionnaire du moyen français $(B T M F)^{16}$.

Nous donnons dans la section suivante nos premiers résultats ; ils concernent selon $X /$ humain/ en ancien français.

\subsection{Selon $\mathrm{X} /$ humain/ en ancien français}

Notre corpus comporte 57 occurrences de ce type; le régime de la préposition est soit un pronom (personnel ou indéfini : 6 exemples), soit un $\mathrm{SN}$; dans ce dernier cas, il s'agit majoritairement d'un nom propre ou d'un SN contenant un nom propre (28 cas).

Nous décrirons d'abord les exemples dans lesquels selon $X$ accepte une des trois interprétations décrites plus haut à propos du FC ; nous verrons ensuite un emploi propre au français médiéval.

\subsubsection{SELON 1 (conformité)}

C'est l'interprétation la plus courante (26 occurrences) ; dans notre corpus, X désigne presque toujours soit Dieu ou Jésus-Christ, soit une personne (ou un groupe de personnes) à qui est attribué un récit ${ }^{17}$; dans ce dernier cas, l'interprétation «conformité » ne se construit que si le verbe lui-même est un verbe de dire :

25. [entre 1172 et 1176] Thomas, Tristan, éd. F. Lecoy, v. 2068 : Entre ceus qui solent cunter / E del cunte Tristran parler, / Il en cuntent diversement : / Oï en ai de plusur gent. / Asez sai que chescun en dit / E ço que il unt mis en escrit, / Mes sulun ço que j'ai oï, / Nel dient pas sulun Breri / Ky solt les gestes e les cuntes / De tuz les reis, de tuz les cuntes / Ki orent esté en Bretaingne. ["Chez tous les conteurs, et plus 


\begin{abstract}
particulièrement chez ceux qui racontent l'histoire de Tristan, il y a des versions différentes. J'en ai entendu plusieurs. Je sais parfaitement ce que chacun raconte et ce qui a été couché par écrit. Mais, d'après ce que j'ai entendu, ces conteurs ne suivent pas la version de Bréri qui connaissait les récits épiques et les contes de tous les rois et de tous les comtes ayant hanté la Bretagne. » Trad. de D. Lacroix et P. Walter, LGF, coll. « Lettres gothiques », 1989, p. 435.]
\end{abstract}

Dans tous les cas, le Gprép. est endophrastique et il constitue le rhème ou en fait partie; on notera que dans l'ex. (25), il est dans la portée de la négation, le procès signifié par « le dient» étant en revanche acquis (le fait que «certains » ont raconté l'histoire de Tristan est affirmé précédemment : «Entre ceus qui solent cunter / E del cunte Tristran parler, / Il en cuntent diversement : / Oï en ai de plusur gent ») ; du point de vue syntaxique, le Gprép. peut fonctionner soit comme complément argumental (il sature donc la valence verbale) - ainsi en (26) où le verbe, soi maintenir « se comporter », nécessite un complément :

26. [entre 1269 et 1278] Jean de Meun, Le roman de la Rose, éd. F. Lecoy, v. 11503 : - (...) Don ne crainz tu pas Dieu ? - Non, certes, / Qu'enviz peut a grant chose ateindre / En cest siecle qui Dieu veust creindre ; / Car li bon, qui le mal eschivent / Et leaument du leur se vivent / Et qui selonc Dieu se maintienent, / Enviz de pain a autre vienent. [ « - Ne crains-tu donc pas Dieu ? - Non, en vérité, car en ce bas monde, celui qui veut craindre Dieu peut difficilement arriver à quelque chose ; en effet, les bons, qui évitent le mal, vivent en toute loyauté de leurs biens et se conduisent selon [les commandements de] Dieu ont bien de la peine à trouver de quoi vivre jour après jour. »]

soit comme adverbial dans la dépendance d'un verbe et de son complément, par exemple dans les exemples (22), (25) (voir supra) et (27) :

27. [entre 1305 et 1309] Joinville, Vie de saint Louis, éd. J. Monfrin, p. 344, § 692 : Quant aucuns benefices de sainte Esglise escheoit [«revenait »] au roy, avant que il le donnast, il se conseilloit a bones personnes de religion et d'autres avant que il le donat. Et quant il s'estoit conseillé, il leur donnoit les benefices de sainte Esglise en bone foy, loialment et selonc Dieu.

soit enfin comme syntagme adjectival (en fonction d'attribut ou d'épithète), comme dans l'ex. (28) où le Gprép. en selon est coordonné par ne à un adjectif :

28. [c. 1283] Philippe de Beaumanoir, Coutumes de Beauvaisis, éd. A. Salmon, p. $129, \S 251$ : Ce n'est pas bon ne selonc Dieu que lonc plet [«procès »] et grant coust soient mis en petites quereles.

Dans cette interprétation «conformité », le régime de selon est en référence indirecte : Dieu réfère à des règles de vie et Breri, à une version particulière (dont il est l'auteur) d'un récit. C'est donc - en partie au moins - à partir de connaissances extralinguistiques que cette interprétation peut se construire.

Tableau 2 : Caractéristiques des occurrences du corpus interprétées comme SELON 1

\begin{tabular}{|l|l|l|}
\hline \multirow{5}{*}{ SELON $X$} & Point de vue sémantique & $\begin{array}{l}\text { Endophrastique : participe à la } \\
\text { création du sens référentiel }\end{array}$ \\
\cline { 2 - 3 } & Point de vue informatif & Rhématique \\
\cline { 2 - 3 } & Point de vue syntaxique & $\begin{array}{l}\text { - Complément argumental du verbe } \\
\text { - Complément adverbial du verbe } \\
\text { - Attribut ou épithète }\end{array}$ \\
\hline \multirow{3}{*}{$X$} & Point de vue morphosyntaxique & $\begin{array}{l}\text { Pronom personnel, SN (souvent } \\
\text { nom propre) }\end{array}$ \\
\cline { 2 - 3 } & Point de vue référentiel & Référence indirecte \\
\hline
\end{tabular}




\title{
3.2.2 SELON 2 (dépendance)
}

Comme dans le cas de SELON 1, le Gprép. est endophrastique et constitue le rhème ou en fait partie. Les exemples sont moins nombreux; dans l'exemple suivant, deux Gprép. en selon avec régime /humain/ sont coordonnés à un Gprép. avec régime /non animé/ :

\begin{abstract}
29. [c. 1283] Philippe de Beaumanoir, Coutumes de Beauvaisis, éd. A. Salmon, p. $429, \S 823:$. ... et la tierce maniere de mesfes [ «méfait»] doit estre vengie par perte d'avoir sans mort et sans mehaing [«blessure corporelle »] et sans prison, et n'est pas l'amende onnie [ «uniforme »] ne que [ "pas plus que»] des autres que nous avons dites dessus, ainçois [ « au contraire »] est l'une grans et l'autre petite selonc le mesfet, et selonc la persone qui mesfet, et selonc la persone a qui l'en mesfet.
\end{abstract}

Ici, la valeur de dépendance se construit dans un énoncé non actuel (énoncé d'une règle juridique) évoquant l'idée de diversité («n'est pas onnie», "l'une grans et l'autre petite») et où $\mathrm{X}$ est un SN singulier mais qui réfère, de façon non spécifique, à une pluralité de référents (les diverses personnes qui commettent un méfait, les diverses victimes) : dans son discours, le locuteur ne réfère pas à des individus particuliers, il renvoie à une sorte de classe intensionnelle définie par la relative.

Le régime de selon peut être un nom propre (coordonné ici à un nom commun) :

30. [c. 1245] La Estoire de Seint Aedward le Rei, éd. K. Y. Wallace, v. 580 : Or sunt Daneis plus fors e pruz, / Ore est meistre reis Hardecnutz, / Solum Fortune e sa riote [《et son fouet»] / K'en guere fait de genz pelote [«jouet»], / Sulum custume de guere, Ore au perdere, ore au cunquere.

La répétition de l'adverbe $\operatorname{or}(e)($ Or...., Ore... « tantôt..., tantôt... ») évoque une alternance de situations favorables et défavorables et en ce sens, selon nous semble relever de la dépendance - le SN référant indirectement aux dispositions changeantes de Fortune vis-à-vis des hommes.

On ajoutera un cas où selon $X$ est à mi-chemin entre la dépendance et la conformité :

31. [c. 1215] The Anglo-Norman Pseudo-Turpin Chronicle of Williame de Briane, éd. I. Short, p. 55: A l'endemayn nostre emperere prist soun counseyl a plus haut barounys de le oost, e comaunda a touz ke il covereysunt les ois de lour chivaus ke il ne veysunt les barberes, e ke il estopassunt les oreyles ke il ne osseynt les tympans. Checun fist soun comaundement soulum sey. [«Le lendemain, notre empereur délibéra avec les plus puissants seigneurs de l'armée et commanda à tous de couvrir les yeux de leurs chevaux afin qu'ils ne voient pas les masques, et de leur boucher les oreilles afin qu'ils n'entendent pas les tambours. Chacun exécuta ses ordres à sa façon. »]

"Checun fist soun comaundement» est un énoncé actuel contenant un verbe d'action, mais l'indéfini checun introduit une idée de diversité et le régime de selon - le réfléchi soi, coréférent à l'indéfini checun - évoque une pluralité, et non un référent particulier, ce qui conduit à une interprétation en SELON 2; toutefois, une glose du type «chacun agit conformément à ses souhaits» (correspondant à SELON 1) parait également acceptable. On a ici un cas de «neutralisation » : les interprétations SELON 1 et SELON 2 «ne s'opposent [pas], mais constituent des valeurs non contradictoires, des chemins interprétatifs résultativement équivalents »; on aboutit ainsi à une « signification intermédiaire ou indistincte » (Fuchs $1988: 14)$.

Tableau 3 : Caractéristiques des occurrences du corpus interprétées comme SELON 2

\begin{tabular}{|l|l|l|}
\hline \multirow{2}{*}{ SELON $X$} & Point de vue sémantique & $\begin{array}{l}\text { Endophrastique : participe à la } \\
\text { création du sens référentiel }\end{array}$ \\
\cline { 2 - 3 } & Point de vue informatif & Rhématique \\
\hline
\end{tabular}




\begin{tabular}{|l|l|l|}
\hline \multirow{3}{*}{$X$} & Point de vue syntaxique & $\begin{array}{l}\text { Complément adverbial du verbe ou } \\
\text { de la phrase }\end{array}$ \\
\hline \multirow{3}{*}{ Point de vue morphosyntaxique } & $\begin{array}{l}\text { - SN (y compris SN comprenant un } \\
\text { nom propre) } \\
\text { - Pronom réfléchi }\end{array}$ \\
\cline { 2 - 3 } & Point de vue référentiel & Ne réfère pas à un particulier \\
\hline
\end{tabular}

\subsubsection{SELON 3 (origine)}

Dans tous nos exemples, selon $X$ est incident à la phrase ; $Y$ peut dans la plupart des cas s'interpréter comme un "rapport de paroles», mais quelques exemples illustrent le type "marqueur d'opinion", d'autres enfin le type « transfert».

Tableau 4 : Caractéristiques des occurrences du corpus interprétées comme SELON 3

\begin{tabular}{|l|l|l|}
\hline \multirow{5}{*}{ SELON $X$} & Point de vue sémantique & $\begin{array}{l}\text { Exophrastique (ne participe pas à la } \\
\text { création du sens référentiel ; } \\
\text { constitue un commentaire du } \\
\text { locuteur) }\end{array}$ \\
\cline { 2 - 3 } & Point de vue informatif & Non rhématique \\
\cline { 2 - 3 } & Point de vue syntaxique & $\begin{array}{l}\text { Incident à la phrase } \\
\text { (éventuellement elliptique) }\end{array}$ \\
\hline \multirow{3}{*}{$X$} & Point de vue morphosyntaxique & $\begin{array}{l}\text { - Pronom personnel } \\
\text { - SN }\end{array}$ \\
\cline { 2 - 3 } & Point de vue référentiel & - Pronom indéfini \\
\hline
\end{tabular}

\section{- SELON 3A-1 « marqueur d'opinion forte »}

Le régime est un pronom personnel référant au locuteur ; ainsi en (32) :

32. [c. 1174] Guernes de Pont-Sainte-Maxence, Saint Thomas, éd. E. Walberg, v. 175: Quant la dame conçut primes l'enfant, sunga / Ke l'eve de Tamise tut' en sun sain entra. / Uns mestres li espunst a cui el le mustra : / "Mult pueples, fist li il, cist eirs guvernera". / Sulunc mei, vives eves en sun ventre porta. [" À peine la dame avait-elle conçu l'enfant qu'elle eut un songe : toute l'eau de la Tamise entrait dans son sein. Elle le raconta à un savant, qui le lui expliqua, disant: "Votre héritier gouvernera de nombreux peuples". D'après moi, elle portait dans son ventre une eau vive. » Trad. de J. Thomas, Louvain/Paris : Peeters, 2002, p. 43].

Après avoir mentionné, sous forme de discours rapporté, l'interprétation que le « maitre » a faite du rêve, le locuteur livre sa propre interprétation. La proposition $p$ est prise en charge au moment de l'énonciation (on passe du plan du récit au plan du discours) et sulunc mei fonctionne comme marqueur d'opinion forte, cette opinion s'opposant à une autre.

\section{- SELON 3A-2 « rapport de parole »}

Ce type d'emploi se trouve par exemple dans des textes «scientifiques» (voir supra, ex. (24), qui figure aussi dans notre corpus) dans lesquels l'auteur s'appuie constamment sur les écrits d' " autorités », ces propos pouvant être introduits dans le texte par selon ou un verbe de dire (cf. Silvi 2003 : 180-181). 
Le SN régime de la préposition a toujours une référence spécifique et désigne toujours une personne (ou un ensemble de personnes) pourvue d'une autorité intellectuelle, morale ou religieuse (le sage ; l'apostle : cf. supra, ex. 21). Il peut dans certains cas s'agir d'un nom de peuple (romaine/gregesse gent), mais le SN réfère alors clairement aux auteurs appartenant à ces peuples (cf. supra, les Latins dans l'ex. 24); de même, quand le SN est un pronom indéfini ou un SN comprenant un déterminant indéfini, il ne peut d'après le contenu de l'énoncé - que référer à des personnes dotées d'une autorité :

33. [1119] Philippe de Thaon, Comput, éd. I. Short, v. 1852 : De [l']an, kil truvat e ki le cummençat Ans est removement / Sulunc alquante gent : / Di le vus en vertéd / Si cum je l'ai truvéd, / Kar chascun an burjunent / Arbres e lur fruit dunent, / E altres crëatures / Tut sulum lur natures. / Mais sulun altre gent / Est dit tut altrement, / Kar quant il ad alees / Trestute[s] les cuntrees / Que de la sus numai / Quant des signes traitai, / Dunc ad sun curs aléd / E tut avirunéd / Tut issi cum l'anels / E cume les cercels ; / E pur ceste raisun / Quë il vait envirun, / Cest nun li emposerent / E annum le appellerent. / E sacez uncore plus / Que li bers Romulus / Primes le contruvad / E cest nun li dunad [«L'an est 'mouvement', selon certaines personnes (....) Mais selon d'autres personnes, il est appelé tout autrement... »]

\section{- SELON 3A-3 « transfert »}

Le régime est alors le nom Dieu. Selon Dieu peut hors contexte recevoir deux interprétations : SELON 1, endophrastique (voir supra, 3.2.1), ou SELON 3. Pour la plupart des exemples, c'est la première qu'on retient : soit la syntaxe ne permet pas d'autre interprétation (le Gprép. sature la valence verbale, est en fonction d'attribut ou de complément du nom, ou encore il est coordonné à un adverbe endophrastique, comme en 27), soit le contexte exclut l'interprétation «origine » : c'est le cas en (22) où Y concerne les faits et gestes d'un individu particulier ; une interprétation SELON 3 serait à la rigueur possible, mais elle supposerait qu'on lise «ses biens departoit» (« il distribuait ce qu'il possédait») comme une façon parmi d'autres envisageables - de décrire l'action faite par celui que désigne le sujet (ce qui pourrait se gloser par « si je me place du point de vue de Dieu, je peux ou dois dire qu'il distribuait ses biens »); ce serait donc une façon particulière de (re)qualifier une certaine action (SELON 3A-3 « transfert »); or, une telle (re)qualification suppose que l'action ait été posée une première fois, ce qui n'est pas le cas dans cet exemple.

En revanche, dans l'exemple suivant (où l'énoncé contient une évaluation), les deux interprétations semblent possibles :

34. [a. 1185] The Hospitallers' Riwle, éd. K. V. Sinclair, v. 1596 : Deu vus cria a sa facture [«image»], / Si vus dona discretiun [«discernement»] / Ke vus eez [ " ayez »] dilectiun [ « amour »], / Primes a lui [si] cum a pere, / E pus pur lui, amez le frere. / Si le vus dit seint Au[gu]stin / Ke sulum Deu fu bon devin [« théologien »].

La relative contenant le Gprép. peut être glosée par «qui fut un théologien dont les qualités sont celles qu'exige Dieu », auquel cas on a un SELON 1 ; il peut l'être aussi par "qui fut un théologien dont on peut dire, si l'on tient compte de ce que Dieu exige, qu'il est bon » : c'est alors à un SELON 3A-3 « transfert» que l'on a affaire. Il y a ici neutralisation entre deux interprétations différentes qui, dans ce contexte, ne conduisent pas à des « solutions référentielles» différentes. (Fuchs 1988 : 12).

\subsubsection{Autre cas}

35. [1320-1324] Chronique de Morée, éd. J. Longnon, p. 111, § 306 : Et quant Sevastocratora [《chef d'armée grecque »] ot ainxi [《ainsi »] gaaignié la bataille et prins [«pris »] le prince Guillerme et son barnage, si fist illeuc tendre son tref [«il fit tendre à cet endroit son pavillon »] et logier son ost et se reposa. ij. jours pour reposer sa gent et pour enfouir les mors, et pour les navrés qui estoient tant que on n'en savoit que faire. Lors fist venir le prince a son tres [«pavillon»] moult honnerablement et lui fist grant reverence, selonc prisonnier. Et après le prince, si honora moult le seignor de Carintaine et puis les autres barons, cescun [ « chacun »] selon la renommée que il avoit. 
Voici comment nous proposons - sans certitude $-d^{\prime}$ 'interpréter cette occurrence ${ }^{18}:$ selon correspondrait à l'une des définitions fournies par le $T L$ (t. IX, 380) «Im Verhältnis zu, für $»^{19}$ (et serait proche du pour que Cadiot 1991 nomme «typico-prédicatif »: Il est grand pour un jockey); le chef d'armée grecque fait au prince - qui est prisonnier - une révérence qui est considérée comme une "grande révérence ", étant donné qu'elle s'adresse à un prisonnier. Si cette interprétation était juste, on pourrait y voir une certaine parenté avec SELON 3:X ne renvoie certes pas à une «origine» et fait partie du contenu asserté (ce n'est pas un commentaire du locuteur), mais il désigne ce que le locuteur prend en compte (une caractéristique d'un actant, ici son statut de prisonnier) pour qualifier le nom révérence de «grande».

\section{Conclusion}

En ancien français, dans les cas examinés ici - selon + régime /humain/ - l'interprétation se construit à partir des paramètres suivants :

- le type de référence réalisé au moyen du SN régi: si la référence est non spécifique, seule l'interprétation « dépendance » (SELON 2) est possible ;

- le contenu de l'énoncé : la présence d'un élément suggérant une variation conduit vers l'interprétation SELON 2 ; dans le cas où le régime est Dieu, seul un contenu d'ordre évaluatif permet une interprétation « origine » (SELON 3$)$;

- les liens sémantiques et syntaxiques entre selon $X$ et le reste de l'énoncé : si selon $X$ ne peut être mis sous la dépendance d'un constituant, si de plus le référent de X n'entretient pas de rapport, dans le monde référentiel, avec les faits dénotés dans le reste de l'énoncé, il sera interprété comme un SELON 3.

Les connaissances extralinguistiques interviennent également : c'est parce que l'on sait que le Dieu des chrétiens est vu comme imposant à l'homme certaines règles de vie que selon Dieu peut être interprété comme un SELON 1 - sans pour autant exclure SELON 3, si le contenu de l'énoncé est évaluatif.

Notons que la place de l'expression dans la phrase ne nous apparait pas comme un critère discriminant: on observe certes que dans plus de la moitié des cas où l'on interpète selon $X$ comme un SELON 3, le Gprép. est en tête (selon $X+$ sujet ou complément $+\mathrm{V})$, mais le Gprép. peut occuper cette même position et relever de la conformité ( $c f$. ex. 22).

Globalement, le selon $X$ des textes d'ancien français semble donc proche de celui du FC; il est en particulier notable qu'il peut, très tôt, fonctionner de façon autonome par rapport au reste de la phrase, fournissant au locuteur la possibilité de commenter son énoncé ou son énonciation. Toutefois, les emplois de selon dans les deux états de langue ne se recouvrent pas exactement : certains effets de sens attestés en ancien français ne le sont plus aujourd'hui (cf. 3.2.4); inversement, selon $+\mathrm{SN}$ désignant un être humain « quelconque » (non pourvu d'une autorité motrale ou savante : Selon la concierge, le voisin du dessus est en vacances) n'est pas attesté en ancien français. La poursuite de l'étude du corpus devra permettre de préciser ces premiers résultats.

\section{Références bibliographiques}

Authier-Revuz, J. (1992). Repères dans le champ du discours rapporté I. L'information grammaticale, 55, 38-42.

Authier-Revuz, J. (1993). Repères dans le champ du discours rapporté II», L'information grammaticale, 56, 10-15.

Borillo, A. (2004). Les adverbes d'opinion forte selon moi, à mes yeux, à mon avis : point de vue subjectif et effet d'atténuation. Langue française, 141, 31-40.

Cadiot, P. (1991). De la grammaire à la cognition. La préposition pour. Paris : Editions. du CNRS.

Charolles, M. (1987). Spécificité et portée des prises en charge en 'selon A'. In Pensée naturelle, logique et langage. Hommage à Jean-Blaise Grize, Université de Neuchâtel, 243-267.

Charolles, M. (1997). L'encadrement des discours. Univers, champs, domaines et espaces. Cahiers de Recherches en linguistique, 6, Nancy : Presses universitaires de Nancy. 
Coltier, D. (2003). Analyse sémantique de selon. Quelques propositions (Présentation de thèse). Travaux de linguistique, 46, 117-131.

Coltier, D. et Dendale, P. (2004). La modalisation du discours de soi : éléments de description sémantique des expressions pour moi, selon moi et à mon avis. Langue française, 141, 41-57.

Combettes, B. (1995). Approche diachronique des adverbiaux contextuels. LINX, 32, 33-50.

Fuchs, C. (1988). Représentation linguistique de la polysémie grammaticale. T. A. Informations, 1-2, 7-20.

Guimier, C. (1996). Les adverbes du français : le cas des adverbes en -ment. Gap/Paris : Ophrys.

Molinier, C. et Lévrier, F. (2000). Grammaire des adverbes : description des formes en -ment. Genève : Droz.

Schrepfer-André, G. (2005). Incidence des formes de reprise du SN régime des SP en selon $X$ énonciatifs sur leur portée phrastique et textuelle. Langue française, 148, 80-94.

Silvi, C. (2003). Science médiévale et vérité. Etude linguistique de l'expression du vrai dans le discours scientifique en langue vulgaire. Paris : Honoré Champion.

Traugott, E. C. (1989). On the rise of espistemic meanings in English : an example of subjectification in semantic change. Language, 65-1, 31-55.

Traugott, E. C. (1995). Subjectification in grammaticalisation. In Stein, D. et Wright, S. (éds.), Subjectivity and subjectivisation in language, Cambridge : Cambridge University Press, 31-54.

\section{Dictionnaires}

AND : Anglo-Norman Dictionary. Sur le site Anglo-Norman On-line Hub : <http://www.anglo-norman.net $>$

FEW : Wartburg, W. von (1963-1989). Französisches etymologisches Wörterbuch. Band XI, Basel : Zbinden.

GD : Godefroy, F. (1880-1902). Dictionnaire de l'ancienne langue française du IX au XVe siècle. Paris : Wieweg et Bouillon.

GLLF : Grand Larousse de la langue française. Paris : Larousse.

Lexis : Dictionnaire de la langue français : Lexis. Paris : Larousse.

NPR : Le Nouveau Petit Robert. Dictionnaire Le Robert, 1993.

$T L$ : Tobler, A. et Lommatzsch, E. (1925-2002). Altfranzösisches Wörterbuch... Wiesbaden/Stuttgart : Franz Steiner.

$T L F i$ : Trésor de la langue française informatisé, Atilf-CNRS : <http://atilf.atilf.fr/tlf.htm $>$

\footnotetext{
${ }^{1}$ Ces travaux portant soit sur l'ensemble des emplois : Coltier 2003, soit sur certains d'entre eux : Borillo 2004, Charolles 1987 et 1997, Coltier et Dendale 2004, Schrepfer-André 2003.

${ }^{2}$ Voici les définitions que Guimier donne de ces notions dans son ouvrage sur la classifications des adverbiaux en ment: "A l'opposition adverbe de constituant / adverbe de phrase, nous substituerons l'opposition adverbe endophrastique / adverbe exophrastique. Les premiers sont, d'un point de vue sémantique, des constituants internes à la phrase, qui affectent le contenu même de l'élément sur lequel ils portent et, ce faisant, participent à la construction du sens référentiel de la phrase. [...]. Les adverbes exophrastiques sont des constituants externes à la phrase, en ce sens qu'ils ne participent pas à la construction de son sens référentiel, mais représentent des traces de l'intervention du locuteur, qui commente tout ou partie de son énoncé ou de l'acte qui le produit. Ils apportent donc des informations qui concernent un ou plusieurs éléments constitutifs de l'acte de d'énonciation (l'énoncé lui-même, l'énonciateur, le co-énonciateur, etc.) » (Guimier (1996:5-6; gras de l'auteur).

3 «L'incidence, notion empruntée au linguiste français Gustave Guillaume, a trait au support syntaxique du mot, à l'unité à laquelle il est rattaché dans la phrase. » (Guimier $1996: 3$; gras de l'auteur). L'incidence, notion syntaxique, est donc clairement distinguée de la portée, qui est en revanche d'ordre sémantique : «la portée [de l'adverbe] concerne sa référence sémantique, c'est-à-dire l'élément à propos duquel l'adverbe dit préférentiellement quelque chose. » (Guimier 1996 : 4) ; un adverbial exophrastique peut ainsi porter sur le dit, le dire ou sur la visée de discours. Nous n'aborderons pas ici la question de la délimitation exacte de la portée des Grprép. en selon.
} 


\begin{abstract}
${ }^{4} \mathrm{X}$ peut être le pronom moi : Voilà mon passage entier, sur lequel votre erreur saute aux yeux. Elle consiste en ce que vous avez entendu ou fait entendre que, selon moi, il falloit avoir été instruit de l' existence de Dieu pour y croire. Ma pensée est fort différente. Je dis qu'il faut avoir l'entendement développé et l'esprit cultivé jusqu'à certain point, pour être en état de comprendre les preuves de l'existence de Dieu, et sur-tout pour les trouver de soi-même sans en avoir jamais entendu parler. (Jean-Jacques Rousseau, Lettre à Christophe de Beaumont, 1763, p. 43, dans Frantext)
\end{abstract}

${ }^{5}$ Le transfert relèverait sans doute, chez Authier-Revuz (1992 et 1993) de la modalisation en discours second.

${ }^{6}$ « Exactly as would be expected, where manner and epistemic (sentential) adverb meanings coexist, the former is earlier than the latter. (...) the meaning based in the sociophysical world precedes that based in the speaker's mental attitude. » (Traugott 1989 : 46).

${ }^{7}$ D'après les exemples fournis par le $T L$ et le $G D$, son/sonc $X$ et lonc $X$ peuvent être glosés tantôt par « conformément à », tantôt par « en fonction de »; une interprétation proche de SELON 3 n'est pas exclue, au moins pour lonc: Lonc le mien sens [«à mon avis»], tot entresait [assurément], Se il l'ëust por autrui fait Que por Pollinicès son frere, Saciés [« sachez-le»], il fust träitre lere [« il aurait été un ignoble traitre »] (Thèbes App. IV 10705, Bd II, $S$ 229, dans $T L$, t. V, 637).

${ }^{8}$ Sauf erreur, ni le Gaffiot, ni l'Oxford Latin Dictionary ne mentionnent pour secundum un emploi du type SELON 3.

${ }^{9} C f$. éd. C. Storey, Genève/Paris : Droz/Minard, 1968, introduction.

${ }^{10}$ Selon est par ailleurs adverbe de lieu en français médiéval (« à côté »).

${ }^{11}$ Au moins selon le $T L F i$; le $F E W$, renvoyant à Palsgrave, date SELON 3 de 1530 ; le GLLF donne également cette date pour SELON 3 + nom de personne, mais place plus tôt l'émergence d'autres emplois correspondant à SELON 3 («Si l'on se réfère à tel principe, à telle manière de penser ou de juger, à tel texte ou à telle oeuvre » v. 1370, Oresme; « si l'on s'en tient à » fin $\mathrm{XV}^{\mathrm{e}}$, Commynes).

${ }^{12}$ Il s'agit en l'occurrence de l'épitre aux Corinthiens de Paul ( $c f$. éd. J. Thomas, t. II, p. 194).

${ }^{13}$ Par ex. : [v. 1181-1185] Chrétien de Troyes, Perceval, v. 39 : La senestre, selonc l'estoire, / Senefie la vainne gloire [«La [main] gauche, dans la tradition, veut dire l'ostentation...» Trad. de C. Méla, LGF, coll. « Pochothèque », 1994, p. 944] ; «C'est que la main gauche - à ce qu'on dit - représente la vaine gloire... » Trad. de J. Ribard, Paris : Honoré Champion, 1983, p. 13]

${ }^{14}$ BFM - Base de Français Médiéval [En ligne]. Lyon : UMR ICAR / ENS-LSH, $2005<\mathrm{http}: / / \mathrm{bfm}$.ens-lsh.fr>. Le site de la $B F M$ n'étant pas consultable actuellement, nous avons travaillé à partir d'une version ancienne (mai 2001) de la base sur cédérom.

15 Adresse : <http://www.anglo-norman.net $>$

${ }^{16}$ ATILF/Nancy Université - CNRS : <http://atilf.atilf.fr/dmf.htm>. Nous avons consulté cette base par le biais de Frantext. (La base est désormais interrogeable par le $D M F^{2}$ ).

${ }^{17}$ Nous relevons par ailleurs une occurrence où le régime est le pronom personnel de $1^{\text {ere }}$ personne : [XIII ${ }^{\mathrm{e}} \mathrm{s}$.] Les Gius Partiz des Eschez. Two Anglo-Norman Chess Treatises, éd. T. Hunt, v. 195 : C'est un guy [« jeu »] sutil et beal, / Si est appellé 'guy de anel'. / Le rey blank mys ad en le eschecker/ Un anel entre luy e soun adverser, / E a le rey neyr graunté le ad, / Si il par force ganier porrad, / E si ad mult bele mestrie / De le garder ke ne le perde mie. / Mes ke [«pourvu que »] solunc mey le juera, / Plegge suy [« je garantis »] ke ne le perdera, / Kar a force ne le put prendre / S'il i ad qe sache defendre.

${ }^{18}$ Il n'est pas exclu qu'il s'agisse d'une erreur du copiste, mais la version française de la Chronique de Morée n'est conservée que dans un mansucrit (éd. Longnon, p. LXXXV).

${ }^{19}$ Par exemple : bielle fourme avoit / Selonc le mal k'ëu avoit (Sone, v. 13094,), ou, sans déterminant: franche et debonaire / Et sage selonc jone ëage (Escan. v. 14645). 